\title{
Actividad investigadora y contexto económico. El caso de las universidades públicas españolas
}

\author{
Teodoro Luque-Martínez* \\ * Facultad de Ciencias Económicas y Empresariales, Universidad de Granada. España \\ Correo-e: tluque@ugr.es
}

Recibido: 17-02-2014; 2a versión: 06-04-2014. Aceptado: 25-04-2014.

Cómo citar este artículo/Citation: Luque-Martínez, T. (2015). Actividad investigadora y contexto económico. El caso de las universidades públicas españolas. Revista Española de Documentación Científica, 38(1): e076. doi: http://dx.doi.org/10.3989/ redc.2015.1.1135

\begin{abstract}
Resumen: La actividad investigadora de las universidades se realiza en un contexto socio-económico y un territorio determinado. Sus dimensiones pueden verse condicionadas por dicho contexto. Este trabajo analiza la actividad investigadora de las universidades públicas presenciales españolas en relación con el PIB per cápita de su territorio, para datos de la primera década del siglo XXI obtenidos del Observatorio IUNE. Se analiza la relación entre PIB y las diferentes dimensiones de la actividad investigadora: reconocimiento, actividad científica, innovación, competitividad, y capacidad formativa. El contexto económico no es neutro y presenta correlaciones significativas, así como relaciones lineales entre el PIB pc y diversas dimensiones. Especialmente, la significación se constata para las dimensiones relativizadas por el número de profesores. En particular para la competitividad, capacidad formativa o actividad científica. En suma, hay diferencias estadísticamente significativas entre las universidades localizadas en los territorios más ricos frente a las de territorios menos ricos.
\end{abstract}

Palabras clave: Indicadores de actividad investigadora; investigación y PIB; $I+D+i$ de la universidad y PIB en España; actividad científica y PIB; competencia académica y PIB; capacidad formativa y PIB.

\section{Research activity and economic context: the case of Spanish public universities}

Abstract: Universities' R\&D and innovation activity takes place within the economic and social context of a specific territory. This activity is multidimensional and its different dimensions may be unduly influenced by the economic context in which it is carried out. The present work analyses the research activity of Spanish public universities in the context of per capita GDP of their territories. The work examines data from 2002-2010 obtained from the IUNE observatory. The relationship between GDP and the different dimensions of research activity are analyzed: recognition, scientific activity, innovation, competitiveness and research training capacity. The results show that the economic context in which the activity takes place is not neutral and that there are significant correlations and linear relationships between per capita GDP and various dimensions of research activity. The significance is particularly notable once the dimensions are relativized according to the number of inhabitants. Statistically significant differences have also been found for different dimensions of research activity between universities located in territories with higher, or lower, per capita GDP.

Keywords: Dimensions of research activity; research and GDP; R\&D and GDP in Spain; scientific activity and GDP; competitiveness and GDP; research training capacity and GDP.

Copyright: ( 2014 CSIC. Este es un artículo de acceso abierto distribuido bajo los términos de la licencia Creative Commons Attribution-Non Commercial (by-nc) Spain 3.0. 


\section{INTRODUCCION}

La actividad investigadora es una de las funciones clave de las universidades, medir su desarrollo y desempeño es trascendental para la gestión de las universidades, tanto en el ámbito interno para orientar su política científica, como en el ámbito externo para orientar las decisiones relativas a I+D+i de los territorios ( Li y otros, 2012; Sun y Liu, 2012; Lin y Roberts, 2012).

Toda investigación se realiza en un contexto determinado. La actividad investigadora de una universidad está condicionada y al mismo tiempo influye en su entorno (Jaffe, 1989; Acs y otros, 2002; OCDE, 2011, Luque-Martínez, y otros 2009). El modelo de la triple hélice que propone la colaboración entre universidad, industria y gobierno (Etzkowitz y Leydesdorff, 1998; Kim y otros, 2012) es un ejemplo de esta interacción. Por un lado, las universidades son un motor de la denominada nueva economía que exige cada vez mayor relación entre las instituciones de educación superior con la industria y las empresas (Olssen y Peters, 2005; Vincett, 2010). El conocimiento y el desarrollo de capital humano constituyen factores de atracción para el sector privado y para los sectores de producción de servicios especializados, en particular. Sin embargo, el papel de las universidades como motores de conocimiento y de desarrollo regional no está confirmado de manera uniforme en los estudios empíricos (Acs y otros, 1997), por tanto, siendo importante como motor, no en todos los casos se ha constatado ese papel de la universidad.

Los estados y los territorios toman conciencia de la importancia estratégica de la actividad investigadora en el desarrollo (King, 2004) e impulsan programas para que ello se traduzca en innovación y desarrollo, en definitiva, en generación de riqueza. Así, la Estrategia Europa 2020 pone un énfasis especial en el apoyo a la $\mathrm{I}+\mathrm{D}+\mathrm{i}$ y al estímulo del crecimiento y promueve estrategias de especialización inteligente en los territorios (RIS-3, 2013), con las universidades como un agente especial.

Por otro lado, el contexto en el que se realiza la actividad investigadora condiciona sus posibilidades. Así, un territorio más dinámico económicamente o con un sistema de $\mathrm{I}+\mathrm{D}+\mathrm{i}$ más desarrollado puede aportar más recursos, plantear retos e iniciativas que contribuyan a generar contratos con los grupos de investigación de las universidades, o a generar patentes o bien proyectos de investigación que concurran a convocatorias nacionales o internacionales con más garantías de éxito, incluso esa conexión con el entorno puede favorecer la elaboración de tesis doctorales. Y no solamente habría que tener en cuenta aspectos económicos sino de funcionamiento, el grado de control político por parte de la administración pública y la elección de los gestores en un territorio determinado tiene influencia sobre las tasas, los precios públicos y los recursos de las universidades (Lowry, (2001).
En los rankings internaciones de universidades más notorios y aceptados (Academic Ranking of World Universities de Shanghai, ARWU (2013), Times Higher Education. THE (2013), National Taiwan University Ranking o Ranking NTU (2013), entre otros), que tienen especialmente en cuenta la actividad investigadora, se comprueba que las universidades que ocupan las primeras posiciones están en territorios de elevado desarrollo económico, son los más ricos y los que tienen mejores sistemas de I+D+i.

Precisamente, el objetivo de este trabajo es analizar la relación entre los resultados de la actividad investigadora de las universidades y el contexto económico, en el que se realiza dicha actividad. Este análisis se circunscribe a las universidades públicas presenciales españolas durante la primera década del siglo XXI. Para analizar esta relación se recurre a los indicadores del Observatorio IUNE (Sanz-Casado, 2012; Sanz-Casado y otros, 2013) diferenciando por sub-dimensiones de la actividad investigadora (reconocimiento, producción, innovación, competitividad o capacidad formativa) y como indicador del contexto económico se recurre al producto interior bruto per cápita del territorio (PIB pc).

\section{MEDIDA DE LA ACTIVIDAD INVESTIGADORA Y DEL CONTEXTO ECONÓMICO}

En España una de las medidas más completas de la actividad investigadora de las universidades es la recogida, desde su aparición en 2012, por el Observatorio IUNE coordinado por el Laboratorio de Estudios Métricos de la Información (LEMI) (Sanz-Casado, 2012; Sanz-Casado y otros, 2013). Se trata de una herramienta de gran valor para nuestros objetivos y que contiene los datos desde el año 2002 agrupados en las dimensiones de la tabla I, y procedente de fuentes oficiales, por lo que son los de más fiabilidad y homogéneos entre los disponibles (Luque-Martínez, 2013). Estas dimensiones comprenden unos cuarenta indicadores de valores absolutos y relativos o referidos al número de profesores, como se describe en la tabla II y referidos a valores totales (suma del periodo) o promedios, señalados por Tot o Prom, o al número de profesores (se indican por 100P).

Como indicador del contexto económico se recurre al producto interior bruto per cápita (PIB pc) del territorio de influencia de la universidad. El PIB es la expresión monetaria de la producción de bienes y servicios de demanda final en un territorio. Ha sido muy utilizado en la contabilidad nacional de todos los países, desde que se impulsó su utilización por el premio Nobel de Economía Kuznets, considerado su inventor, entre otros economistas. Es una medida macroeconómica de la producción material en un espacio y tiempo determinado, es una medida recogida en la Contabilidad Regional de España elaborada por el Instituto Nacional de Estadística (INE, 2012). Si el PIB de un territorio se divide por el número de habitantes de dicho espacio se obtiene el PIB per cápita (PIB pc). 
Tabla I. Dimensiones de la actividad investigadora y fuentes de información. Fuente: http://www.iune.es/

\begin{tabular}{ll}
\hline Dimensiones de la Actividad Investigadora & Fuente de información \\
\hline Reconocimiento & $\begin{array}{l}\text { CNEAI (Comisión Nacional de Evaluación de la Actividad } \\
\text { Investigadora) / Ministerio de Ciencia e Innovación*. }\end{array}$ \\
\hline Actividad científica & $\begin{array}{l}\text { Plataforma Web of Science (Science Citation Index, Social Science } \\
\text { Citation Index, y Arts \& Humanities Citation Index). }\end{array}$ \\
\hline Innovación & $\begin{array}{l}\text { Red OTRI (Encuesta anual a las universidades) / } \\
\text { INVENES (creada por la Oficina Española de Patentes y Marcas). }\end{array}$ \\
\hline Competitividad & $\begin{array}{l}\text { CDTI (Centro para el Desarrollo Técnico Industrial) / MICINN } \\
\text { (Ministerio de Ciencia e Innovación*). }\end{array}$ \\
\hline Capacidad formativa & Ministerio de Educación / INE (Instituto Nacional de Estadística). \\
\hline Actualmente el Ministerio de Educación se denomina Ministerio de Educación, Cultura y Deporte, mientras que el Ministerio de \\
Ciencia e Innovación ha desaparecido y sus funciones las asume el Ministerio de Economía y Competitividad.
\end{tabular}

Tabla II. Descripción de los indicadores de la actividad investigadora. Fuente: Observatorio IUNE

\begin{tabular}{|c|c|}
\hline Etiquetas & Descripción \\
\hline \multicolumn{2}{|r|}{ Reconocimiento } \\
\hline Sex_100Prof & Número de sexenios obtenidos por universidad por cada 100 profesores \\
\hline \multicolumn{2}{|r|}{ Actividad científica } \\
\hline Publ_Prom & Publicaciones por universidad y año \\
\hline PubxProf_Prom & Publicaciones por profesor, universidad y año \\
\hline Coauto_Prom & Autores por documento de la producción científica de cada universidad cada año \\
\hline Colabinter_Prom & $\begin{array}{l}\text { Porcentaje de documentos firmados por cada universidad con, al menos, una } \\
\text { institución extranjera, respecto al total de documentos firmados por cada universidad } \\
\text { cada año }\end{array}$ \\
\hline Citas_Tot & Número de citas recibidas por la producción científica anual de cada universidad \\
\hline CitasProf_Prom & Ídem por profesores \\
\hline Docno_Prom & $\begin{array}{l}\text { Porcentaje de documentos por universidad y año, que desde su publicación hasta el } \\
\text { momento de actualización de los datos, no han recibido ninguna cita }\end{array}$ \\
\hline 1C_S_Tot & Publicaciones en revistas del 1er cuartil (total) \\
\hline 1C_Prom & Publicaciones en revistas del 1er cuartil (promedio) \\
\hline Top3_Tot & Publicaciones en las 3 primeras revistas de cada una de las áreas científicas (total) \\
\hline Top3_\%Tot & $\begin{array}{l}\text { Porcentaje de publicaciones en las } 3 \text { primeras revistas de cada una de las áreas } \\
\text { científicas }\end{array}$ \\
\hline \multicolumn{2}{|r|}{ Innovación } \\
\hline PatNac_Tot & Número de patentes nacionales (total) \\
\hline PatNac_100P_Tot & Número de patentes nacionales por cada 100 profesores \\
\hline Con_I+D+cons_Tot & Importe de los contratos de I+D y consultorías (total miles euros) \\
\hline $\begin{array}{l}\text { Con_I+D+cons_100P_mi- } \\
\text { les euros_Prom }\end{array}$ & Ídem por cada 100 profesores \\
\hline Pservic_miles_euros & Importe facturado por prestación de servicios (total miles de euros) \\
\hline $\begin{array}{l}\text { Pservic_100P_miles_eu- } \\
\text { ros_Prom }\end{array}$ & Ídem por cada 100 profesores \\
\hline IngLic_miles_euros_Prom & $\begin{array}{l}\text { Importe generado por las actividades de uso, explotación, modificación, etc., (bajo } \\
\text { unas determinadas condiciones, según se acuerde en el contrato de licencia), de una } \\
\text { determinada tecnología o conocimiento de la universidad (total miles euros) }\end{array}$ \\
\hline $\begin{array}{l}\text { IngLic_100P_miles_eu- } \\
\text { ros_Prom }\end{array}$ & Ídem por cada 100 profesores \\
\hline PCT_Prom & $\begin{array}{l}\text { Número de protecciones de invenciones de forma simultánea en distintos países, a } \\
\text { través de la presentación de una solicitud internacional de patentes }\end{array}$ \\
\hline
\end{tabular}




\begin{tabular}{|c|c|}
\hline Etiquetas & Descripción \\
\hline PCT_100P_Prom & Ídem por cada 100 profesores \\
\hline Spinoff_Tot & Número de spin-off creadas (total) \\
\hline Spinoff_100P_Prom & Ídem por cada 100 profesores \\
\hline \multicolumn{2}{|r|}{ Competitividad } \\
\hline Plann_Tot & Número de proyectos obtenidos en convocatorias del Plan Nacional (total) \\
\hline Plann_Prom & Número de proyectos obtenidos en convocatorias del Plan Nacional (promedio) \\
\hline Plann_100P_Tot & $\begin{array}{l}\text { Número de proyectos obtenidos en convocatorias del Plan Nacional por cada } 100 \\
\text { profesores }\end{array}$ \\
\hline PMarco_Tot & Número de proyectos obtenidos en convocatorias del Programas Marco (total) \\
\hline PMarco_Prom & Número de proyectos obtenidos en convocatorias del Programas Marco (promedio) \\
\hline PMarco_100P_Prom & $\begin{array}{l}\text { Número de proyectos obtenidos en convocatorias del Programas Marco por cada } 100 \\
\text { profesores (promedio) }\end{array}$ \\
\hline \multicolumn{2}{|r|}{ Capacidad formativa } \\
\hline FPI_Prom & Número de becas FPI (promedio) \\
\hline FPI_100P_Prom & Número de becas FPI por cada 100 profesores (promedio) \\
\hline FPU_Prom & Número de becas FPU (promedio) \\
\hline FPU_100P_Prom & Número de becas FPU por cada 100 profesores (promedio) \\
\hline JCierva_Prom & $\begin{array}{l}\text { Número de contratos del Programa Juan de la Cierva (contratos de doctores - } 3 \text { años- } \\
\text { para incorporación a equipos de investigación) por universidad (promedio) }\end{array}$ \\
\hline JCierva_100P_Prom & Ídem por cada 100 profesores y universidad (promedio) \\
\hline RyCajal_Prom & $\begin{array}{l}\text { Número de contratos del Programa Ramón y Cajal (contrato laboral doctores - } 5 \text { años- } \\
\text { para incorporación a equipos de investigación) por universidad (promedio) }\end{array}$ \\
\hline RyCajal_100P_Prom & Ídem por cada 100 profesores y universidad (promedio) \\
\hline Tesis_Prom & Tesis doctorales defendidas por universidad (promedio) \\
\hline Tesis_100P_Prom & Tesis doctorales defendidas por cada 100 profesores y universidad (promedio) \\
\hline
\end{tabular}

EI PIB no está exento de críticas, desde luego no es apropiado interpretarlo como medida de bienestar general, porque se trata de una medida de la producción de bienes y servicios. Esto ya lo puso de relieve Kuznets y también Stiglitz y otros (2013). Por otro lado, el PIB per cápita no proporciona información sobre la desigualdad del reparto de la producción entre la población, que puede ser muy desigual o no. No obstante, es un indicador con gran aceptación y muy utilizado como referente del nivel económico de un país y el más asequible para determinados niveles territoriales. En suma, se puede considerar una aproximación aceptable del contexto económico de un territorio.

\section{METODOLOGÍA}

Como se indica en la web IUNE (http://www. iune.es/), este observatorio es una herramienta que permite conocer los resultados más significativos de la actividad científica de las universidades españolas públicas y privadas. En el presente estudio se considera el período 2002-2010 y todas las universidades públicas presenciales, que son 47. Se trabaja con valores medios de la actividad investigadora del período aunque en algunas ocasiones se utilizan valores totales. Para la dimen- sión de "innovación" solamente se disponen de datos desde 2006 a 2010 (exceptuando los indicadores sobre patentes que están completos), por lo que la media del período se calcula con los cinco últimos años de la década. La consulta de los datos IUNE tuvo lugar en mayo 2012. En España, algunas de estas medidas se obtienen mediante la encuesta a la RED OTRI que deben proporcionar las universidades para hacerlas públicas, y esto no siempre ocurre.

Los datos relativos a innovación suelen plantear dificultades adicionales, su medida no está tan resuelta y se tiene menos tradición en la recogida. El caso es que para uno o más indicadores faltaban datos que afectaban a una quincena de universidades, de esta manera el análisis se reducía a un número de casos bastante inferior. Para tratar de solventar esta situación se ha procedido a estimar los valores mediante un procedimiento disponible en el programa LISREL, Ilamado imputación múltiple mediante el algoritmo EM (Jöreskog y Sörbom, 2006). Éste es un procedimiento robusto, mediante el cual se imputan los datos que faltan teniendo en cuenta el perfil de todos los datos disponibles de cada universidad. Parte del supuesto de que la probabilidad de que falte un dato es independiente de 
los valores de la misma variable, pero está relacionada con los valores de las otras variables (Medina y Galván, 2007; Jöreskog y Sörbom, 2006).

Los indicadores de innovación cada vez se tienen más en cuenta, a la vista de la tendencia que pone el énfasis en la protección y apropiación de la producción científica más que en la producción y la difusión de la misma (Tijssen, 2004) por lo que es una dimensión al alza dentro de la actividad investigadora. Al aumentar el interés por la protección se mejora la recogida de indicadores.

Puesto que hay universidades con centros universitarios en una o en más provincias con diferentes niveles de PIB pc, es necesario decidir el PIB que se toma. Pues bien, cuando la universidad esté localizada en una provincia solamente, el PIB que se toma es el de dicha provincia. Si la universidad cuenta con centros en más de una provincia se toma el PIB de la provincia en donde radica la mayor parte de su actividad. Por último, si es una universidad cuya influencia se extiende por toda la Comunidad Autónoma se toma el PIB pc de dicha Comunidad Autónoma. Es el caso de la Universidad de Zaragoza en Aragón o de las universidades de Castilla-La Mancha, Extremadura o País Vasco. Obviamente, las universidades en Comunidades Autónomas uniprovinciales no presentan problema porque el PIB de la Comunidad y de la provincia es el mismo.

El PIB per cápita, ya sea el provincial o el autonómico, se obtiene del Instituto Nacional de Estadística y se refiere a euros de 2010.

Para tener en cuenta el contexto económico del entorno en el que la universidad lleva a cabo su actividad y su relación con la $\mathrm{I}+\mathrm{D}+\mathrm{i}$, se puede proceder de varias formas. Una es hacer un análisis de la actividad investigadora recurriendo a un solo indicador, opción que de momento no se considera a la vista de la complejidad del concepto. Otra opción es desglosar la actividad investigadora en tantos indicadores como existan, lo que resulta poco operativo cuando se quiere ver la posición de cada universidad. Finalmente, se puede optar por una posición intermedia, que es la que ahora se aborda. Concretamente, lo que se persigue es ver la relación existente entre las principales sub-dimensiones que componen el concepto de actividad investigadora y el contexto económico en el que desarrollan su actividad.

La relación de las universidades analizadas, sus acrónimos, antigüedad, tamaño y los PIB pc de los territorios de la provincia o Comunidad Autónoma de referencia (media del período analizado) se presentan en la tabla del anexo 1.

Una vez obtenidos los datos de estas variables se procede de la siguiente manera, para cada dimensión de la actividad investigadora se realiza un análisis de componentes principales (LuqueMartínez, 2012) con el objeto de identificar las componentes que concentran la mayor parte de la información de la dimensión correspondiente. Es decir, se obtienen sub-dimensiones o componentes principales para cada dimensión inicial, que sintetizan o resumen la casi totalidad de la información de partida, y sobre las que se realiza un análisis de regresión, considerando cada componente como la variable dependiente y como variable independiente el PIB per cápita.

Tras todo ello, se lleva a cabo un análisis de la varianza para comprobar la existencia o no de diferencias estadísticamente significativas entre las universidades en territorios con mayor PIB per cápita frente a las que están en los de menor PIB per cápita respecto de las diferentes subdimensiones identificadas de la actividad investigadora. Para todos estos análisis se ha utilizado los siguientes programas SPSS 20, STATISTICA 7 para los análisis, EXCEL para las representaciones gráficas y LISREL para la imputación de datos faltantes.

\section{ANÁLISIS}

Los análisis referidos se aplican para cada una de las diferentes dimensiones que se especifican en el Observatorio IUNE (Sanz-Casado, 2012; Sanz-Casado y otros, 2013), salvo en el caso de "reconocimiento" que no procede el análisis de componentes principales, análisis que se recogen en la tabla III y más adelante se comentan.

\section{Reconocimiento}

Esta dimensión se compone de dos indicadores uno es el número de sexenios por profesor y otro es el número de premios nacionales de investigación. Debido a que en este segundo caso hay frecuencias solamente para un número reducido de universidades, no resulta pertinente la inclusión en el análisis, y solamente se toma en consideración el primer indicador. El número de sexenios de investigación por profesor no está correlacionado con el PIB per cápita territorial a la vista de su bajo y no significativo índice de correlación, cuyo valor es 0,01 . El que la universidad desarrolle su actividad en un territorio con más o menos riqueza económica no tiene relación con el número de sexenios de sus profesores. Tampoco resulta significativa la relación lineal entre el número de sexenios y el PIB pc, ni hay diferencias significativas entre las universidades situadas en territorios con mayor frente a las de menor PIB pc respecto al número de sexenios.

\section{Actividad científica}

La dimensión "actividad científica" comprende todos los indicadores relativos a producción científica, colaboración con otros autores, impacto y visibilidad de la producción científica, en total una docena de indicadores. 
Al aplicar análisis de componentes principales, se comprueba que tres de esas variables no están bien representadas en la estructura de correlación de esta dimensión por lo que deben ser excluidas del análisis. Efectivamente, tales variables tienen características diferentes al resto, no tienen relación con el resto. Esto lo deja muy claro el valor de la comunalidad de cada una de ellas, esto aconseja no contemplarlas en el análisis. De alguna manera sus nombres ya ayudan a comprender su escasa relación con las otras variables de esta dimensión. Son las siguientes: número de autores por documento de la producción científica de cada universidad cada año (promedio), documentos en colaboración nacional y porcentaje de documentos por universidad y año que no han recibido ninguna cita (promedio). El análisis, con un índice $\mathrm{KMO}$ de 0,68 y siendo significativo el test de esfericidad de Bartlett, presenta buenos indicadores para realizar el análisis de componentes principales. Todas las variables observadas tienen una comunalidad elevada, superan el $80 \%$, salvo una que tiene un $74 \%$ (tabla III).

Tabla III. Resumen de los análisis de componentes principales de la actividad investigadora

\begin{tabular}{lccccc}
\hline Dimensión & No variables & $\begin{array}{c}\text { \% varianza } \\
\text { explicada }\end{array}$ & No componentes & $\begin{array}{c}\text { Índice KMo } \\
\text { Prueba de } \\
\text { Bartlett }\end{array}$ \\
\hline Actividad científica & 9 & 84,79 & 2 & 0,68 & 0,00 \\
\hline Innovación & 12 & 75,4 & 3 & 0,69 & 0,00 \\
\hline Competitividad & 4 & 86,18 & 2 & 0,36 \\
\hline Capacidad formativa & 10 & 88,12 & 2 & 0,63 & 0,00 \\
\hline
\end{tabular}

Se han obtenido dos componentes que resumen el $84,8 \%$ de la información. La primera se refiere a magnitudes absolutas de publicaciones, citas o artículos en revistas que ocupan las primeras posiciones (tabla IV), mientras que la segunda componente sintetiza esas variables pero relativizadas por el número de profesores más, y esto es lo llamativo, la variable que registra la colaboración internacional. La colaboración internacional se encuentra asociada a la actividad científica por profesor. Mientras que el número de autores estaba mal representada en este análisis y tenía una baja comunalidad, la colaboración internacional (promedio) si tiene más relación con el número de publicaciones y la calidad de las mismas relativizadas por el número de profesores (medida por número de citas o artículos en el top3 o del primer cuartil).
Es lógico que colaboración internacional, productividad y calidad estén correlacionadas.

La componente primera, que por los indicadores que resumen puede denominarse "actividad científica general", no tiene relación significativa con el PIB pc territorial.

Por otro lado, la segunda componente, que podemos denominar "actividad científica relativa o por profesor", está correlacionada positiva y significativamente con el PIB pc, por lo que a mayor PIB del territorio donde se inserta la actividad de la universidad mayor actividad científica por profesor. En este caso, en el análisis de regresión lineal resulta cuasisignificativa la influencia del PIB pc sobre la actividad científica por profesor, a un nivel del 0,07 , con un $R^{2}=0,07$ y un coeficiente estandarizado beta $=0,27$.

Tabla IV. Matriz de componentes rotados dimensión "actividad científica"

\begin{tabular}{lcc}
\hline & Componente 1 & Componente 2 \\
\hline Publ_Prom & $\mathbf{0 , 9 8 5}$ & 0,133 \\
\hline PubxProf_Prom & 0,147 & $\mathbf{0 , 8 5 7}$ \\
\hline Colabinter_Prom & 0,377 & $\mathbf{0 , 7 3 9}$ \\
\hline Citas_Tot & $\mathbf{0 , 9 6 4}$ & 0,246 \\
\hline CitasProf_Prom & 0,213 & $\mathbf{0 , 9 1 9}$ \\
\hline 1C_S_Tot & $\mathbf{0 , 9 7 6}$ & 0,21 \\
\hline 1C_Prom & 0,243 & $\mathbf{0 , 7 6 3}$ \\
\hline Top3_Tot & $\mathbf{0 , 9 5 5}$ & 0,272 \\
\hline Top3_\%Tot & 0,051 & $\mathbf{0 , 8 3 3}$ \\
\hline
\end{tabular}

Método de rotación: Normalización Varimax con Kaiser. 
Como muestra la figura 1 , entre los territorios con más PIB pc destacan por actividad científica por profesor, las universidades catalanas, muy especialmente la UPF, la Autónoma de Madrid y la Jaume I. Mientras que en los territorios con menor PIB pc lo hacen las universidades de Córdoba, Pablo de Olavide, Huelva, Miguel Hernández o Santiago de Compostela. Lógicamente, todas estas universidades mencionadas están muy por encima de la recta de regresión que marca la tendencia media.

Figura 1. Representación de las universidades públicas españolas según el PIB per cápita territorial y la dimensión "actividad científica por profesor" (los ejes de la figura se han escalado con el fin de ganar en la visibilidad de los puntos)

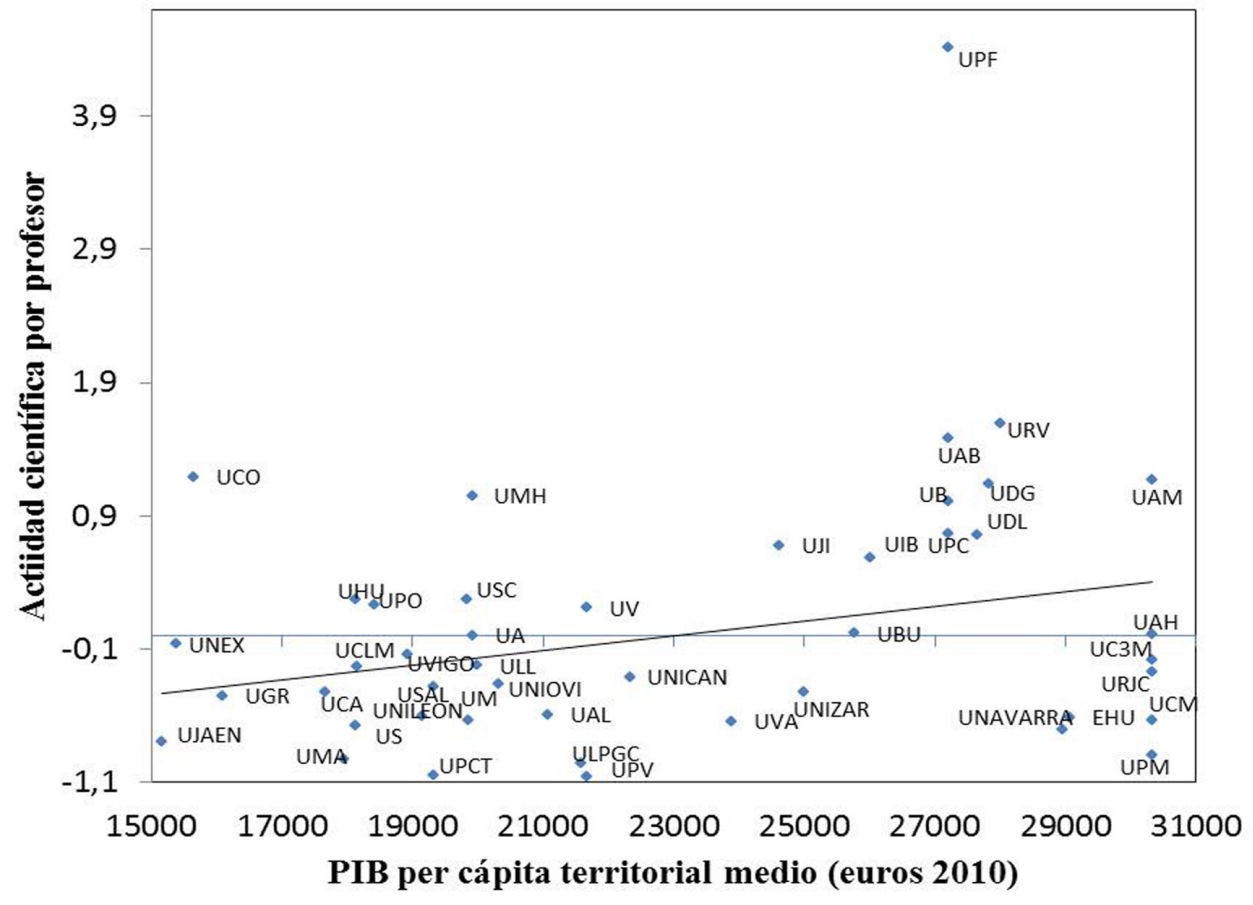

\section{Innovación}

Esta dimensión comprende doce indicadores de la tabla II, relativos a ingresos por contratos de I+D y consultorías y por licencias, al número de patentes y de spin-off. Con la dimensión innovación se procede de igual forma pero es necesario hacer una advertencia: faltan indicadores de esta dimensión en diferentes universidades para diversos años. Por tanto, no es posible tener las medias para la década analizada de diversas universidades. No obstante como ya ha sido comentado, se ha procedido a imputar los valores mediante un procedimiento disponible en el programa LISREL.

El análisis de componentes principales presenta buenos indicadores, con un índice KMO de 0,69 y siendo significativo el test de esfericidad de Bartlett. Todas las variables observadas tienen una comunalidad elevada, superan el $50 \%$ y la mayoría supera el $70 \%$ (tabla III).

Se obtienen tres componentes que concentran más del $75 \%$ de la información de todos los indicadores iniciales. Según la tabla V, la primera componente concentra la información de las variables siguientes: número de patentes, importe de los contratos de I+D y consultorías, importe de lo facturado por prestación de servicios (en valores absolutos y por profesor), número de protecciones de invención de forma simultánea en distintos países. Se puede denominar "patentes e ingresos por contratos de I+D, servicios y patentes".

Esta componente se correlaciona de forma positiva con el PIB pc territorial. Efectivamente, en el análisis de regresión muestra que hay una relación significativa $(0,04)$, para un $R^{2}=0,09$ y un coeficiente estandarizado beta $=0,3$.

La segunda componente claramente reúne la información sobre los ingresos por licencias de uso, explotación, modificación, etc. de determinadas tecnologías o conocimiento, tanto en valores absolutos como por profesores, además del número de protecciones de invención de forma simultánea en distintos países. Esta componente no se correlaciona ni es significativa su relación con el PIB pc.

La tercera componente se identifica con las "spin-off" creadas, tanto en valores totales como relativos por profesor, los ingresos por contratos de $\mathrm{I}+\mathrm{D}$ y consultoría por profesor y el número de patentes por profesor. Es una componente que tiene 
que ver con el emprendimiento. Esta componente no se correlaciona con el PIB y la regresión lineal tampoco resulta significativa.

Con las reservas relativas a esta dimensión, ya mencionadas, y como se representa en la figura 2 , las universidades de territorios con más PIB pc logran más patentes e ingresos por contratos de I+D-consultorías, servicios y los derivados de la protección. En concreto entre las de mayor PIB pc sobresalen las universidades Politécnica de Madrid, de forma destacada, y Complutense de Madrid, Autónoma de Barcelona, Zaragoza y Politécnica de Cataluña y Barcelona; siguen las universidades de la ciudad de Valencia (UPV y UV) y Santiago. En la parte baja del PIB pc sobresalen en esta dimensión las universidades de Extremadura, Sevilla, Córdoba, Castilla-La Mancha y Granada. Todas ellas claramente por encima de la recta de regresión.

Tabla V. Matriz de componentes rotados dimensión "innovación"

\begin{tabular}{lccc}
\hline & Componente 1 & Componente 2 & Componente 3 \\
\hline PatNac_Tot & $\mathbf{0 , 5 9 3}$ & 0,445 & 0,369 \\
\hline PatNac_100P_Tot & 0,085 & 0,491 & $\mathbf{0 , 5 4 1}$ \\
\hline Con_I+D+cons_Tot & $\mathbf{0 , 8 9 7}$ & 0,147 & 0,266 \\
\hline Con_I+D+cons_100P_miles euros_Prom & 0,483 & 0,221 & $\mathbf{0 , 5 2 3}$ \\
\hline Pservic_miles_euros & $\mathbf{0 , 9 3 2}$ & 0,16 & 0,091 \\
\hline Pservic_100P_miles_euros_Prom & $\mathbf{0 , 9 3 3}$ & $-0,008$ & 0,13 \\
\hline IngLic_miles_euros_Prom & 0,393 & $\mathbf{0 , 8 7 1}$ & $-0,011$ \\
\hline IngLic_100P_miles_euros_Prom & $-0,037$ & $\mathbf{0 , 8 7 2}$ & 0,027 \\
\hline PCT_Prom & $\mathbf{0 , 6 7 1}$ & 0,478 & 0,118 \\
\hline PCT_100P_Prom & 0,247 & $\mathbf{0 , 5 0 5}$ & 0,463 \\
\hline Spinoff_Tot & 0,504 & 0,038 & $\mathbf{0 , 7 3 4}$ \\
\hline Spinoff_100P_Prom & 0,014 & $-0,061$ & $\mathbf{0 , 9 3 7}$ \\
\hline
\end{tabular}

Método de rotación: Normalización Varimax con Kaiser.

Figura 2. Representación de las universidades públicas españolas según el PIB per cápita territorial y la dimensión "patentes e ingresos por contratos de I+D, servicios y patentes" (los ejes de la figura se han escalado con el fin de ganar en la visibilidad de los puntos)

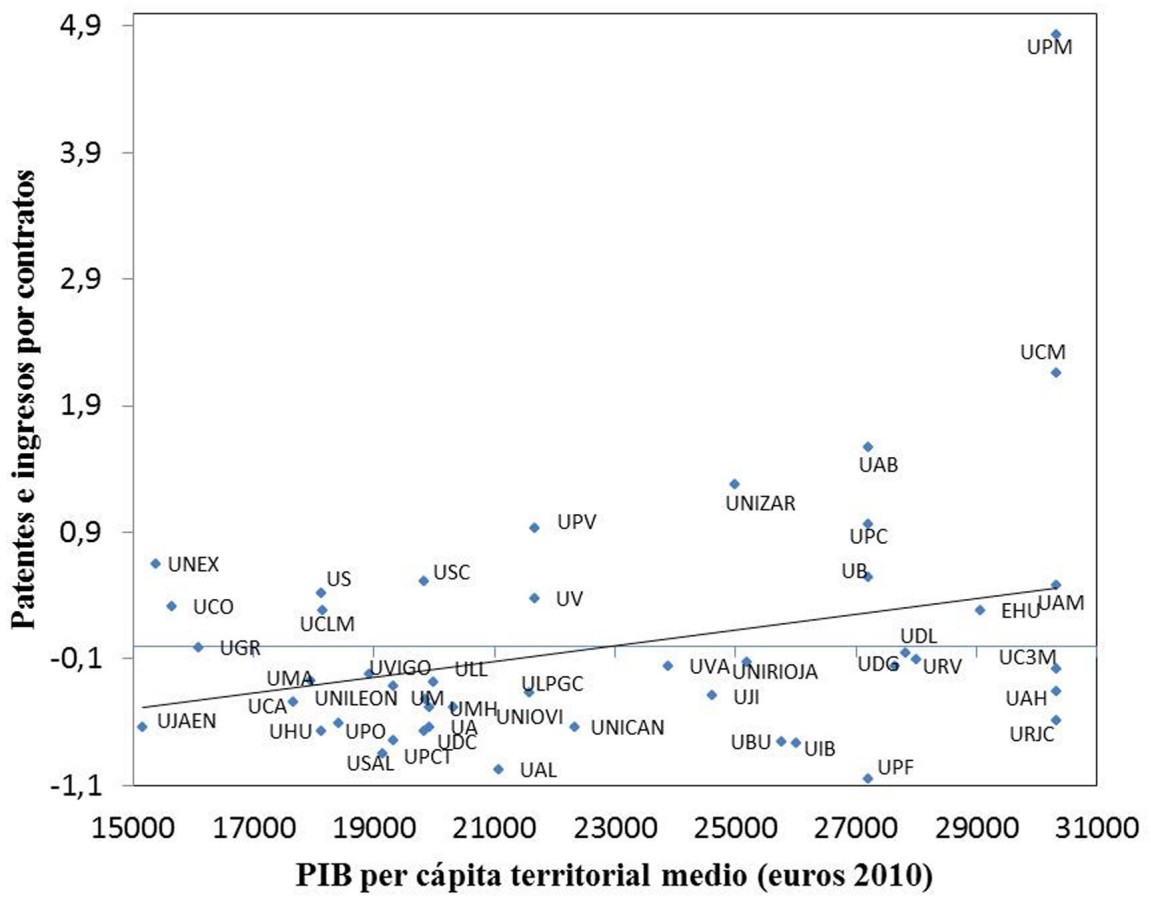




\section{Competitividad}

La dimensión competitividad abarca variables relativas al número de proyectos conseguidos en convocatorias del Plan Nacional y del Programa Marco, tanto en valores totales como promedios o por cada cien profesores, en total cuatro variables. No obstante, se excluye el número promedio de proyectos, tanto del Plan Nacional como del Programa Marco, por ser redundantes con el número de proyectos totales en los respectivos casos (Plan Nacional y Programa Marco), por tanto, el análisis queda con cuatro variables.

El análisis de componentes principales presenta un KMO bajo; pero la prueba de esfericidad es significativa y todas las variables consideradas tienen comunalidades muy elevadas, por encima del $83 \%$ (tabla III). Se han obtenido dos componentes que explican el $86,18 \%$ de la varianza, resultado obvio a la vista de las variables observadas. La primera componente hace referencia al número de proyectos totales conseguidos tanto en el Plan Nacional como en Programas Marco relativizado por número de pro- fesores, mientras que la segunda hace referencia a lo mismo pero en valores absolutos (tabla VI).

La primera componente, que podemos denominar "competitividad relativa o por profesor", presenta correlación con el PIB pc, de manera que cuanto mayor es PIB pc del territorio de influencia de la universidad, mayor es la captación de proyectos del Plan Nacional y de los Programas Marco por número de profesores. La competitividad relativa es explicada de manera significativa $(0,01)$ con un $R^{2}=0,13$ y un coeficiente estandarizado beta de 0,36 por el PIB pc del territorio de la universidad.

De acuerdo con la figura 3, en los territorios con más PIB pc hay que señalar con mucha diferencia a la universidad Pompeu Fabra seguida a distancia de la Carlos III, para continuar con la mayoría de las universidades catalanas (Politécnica de Cataluña, Rovira i Virgili, Autónoma de Barcelona) y también la de Cantabria. En zonas con menor PIB pc sobresalen las universidades Pablo de Olavide y Miguel Hernández, todas muy por encima de la recta de regresión.

Tabla VI. Matriz de componentes rotados dimensión "competitividad"

\begin{tabular}{lcc}
\hline & Componente 1 & Componente 2 \\
\hline Plann_Tot & $-0,055$ & $\mathbf{0 , 9 2 3}$ \\
\hline Plann_100P_Tot & $\mathbf{0 , 9 1 2}$ & 0,004 \\
\hline PMarco_Tot & 0,314 & $\mathbf{0 , 8 7 5}$ \\
\hline PMarco_100P_Prom & $\mathbf{0 , 9 2 1}$ & 0,214 \\
\hline
\end{tabular}

Método de rotación: Normalización Varimax con Kaiser.

Figura 3. Representación de las universidades públicas españolas según el PIB per cápita territorial y la dimensión "competitividad por profesor" (los ejes de la figura se han escalado con el fin de ganar en la visibilidad de los puntos)

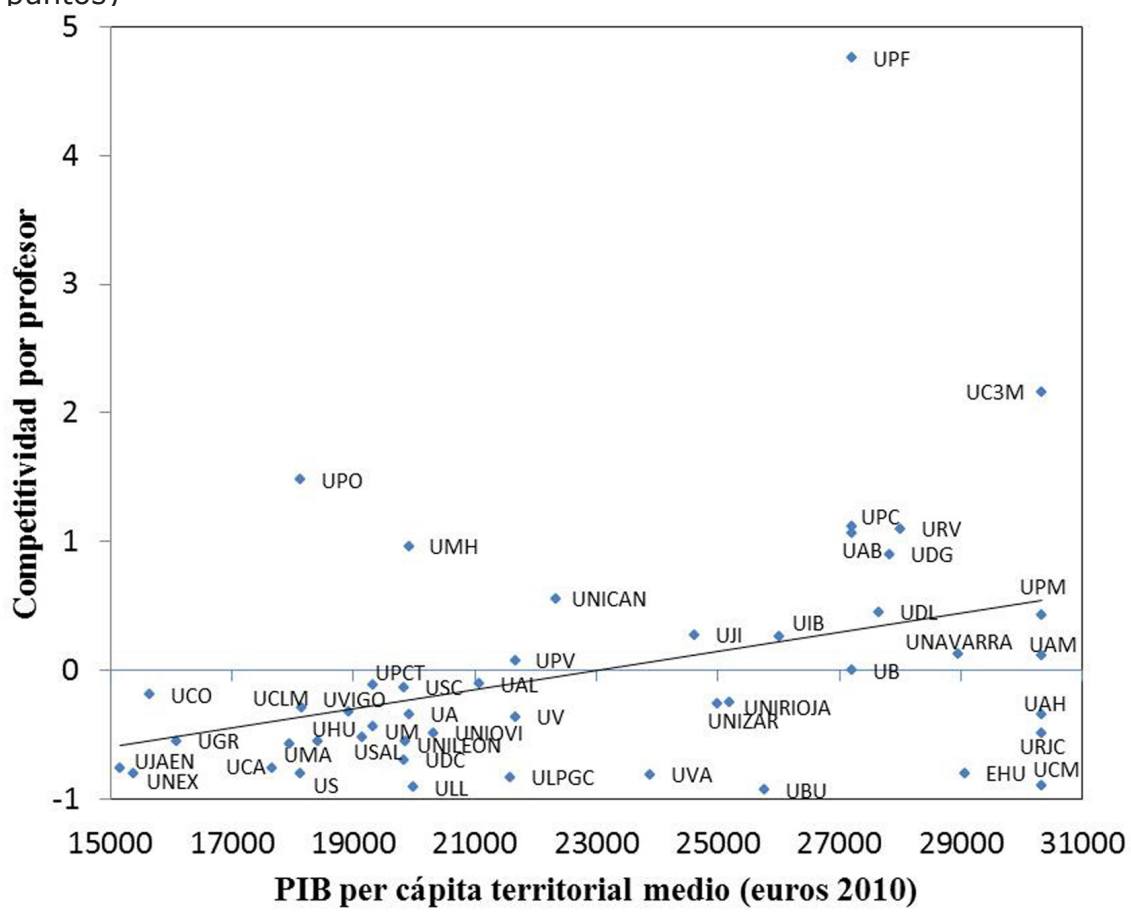


La "competitividad general", segunda componente, presenta correlación estadísticamente significativa con el PIB pc territorial. Esto es, a mayor PIB pc del territorio también es mayor el número de proyectos que se consiguen, ya sean nacionales o europeos. Esta relación es significativa $(0,02)$, con un $R^{2}=0,11$ y un coeficiente estandarizado beta de 0,33.

En esta dimensión, y en términos generales, destacan las universidades grandes. Como se repre- senta en la figura 4, en la parte alta del PIB pc están las universidades de Barcelona, Politécnica y Complutense de Madrid, Autónoma de Barcelona, Politécnica de Cataluña; en el siguiente tramo de PIB están las de Zaragoza, Valencia y Politécnica de Valencia; finalmente, en las zonas de menor PIB pc destacan las universidades de Granada, Sevilla, Santiago y Oviedo, todas superando con creces la recta de regresión.

Figura 4. Representación de las universidades públicas españolas según el PIB per cápita territorial y la dimensión "competitividad general" (los ejes de la figura se han escalado con el fin de ganar en la visibilidad de los puntos)

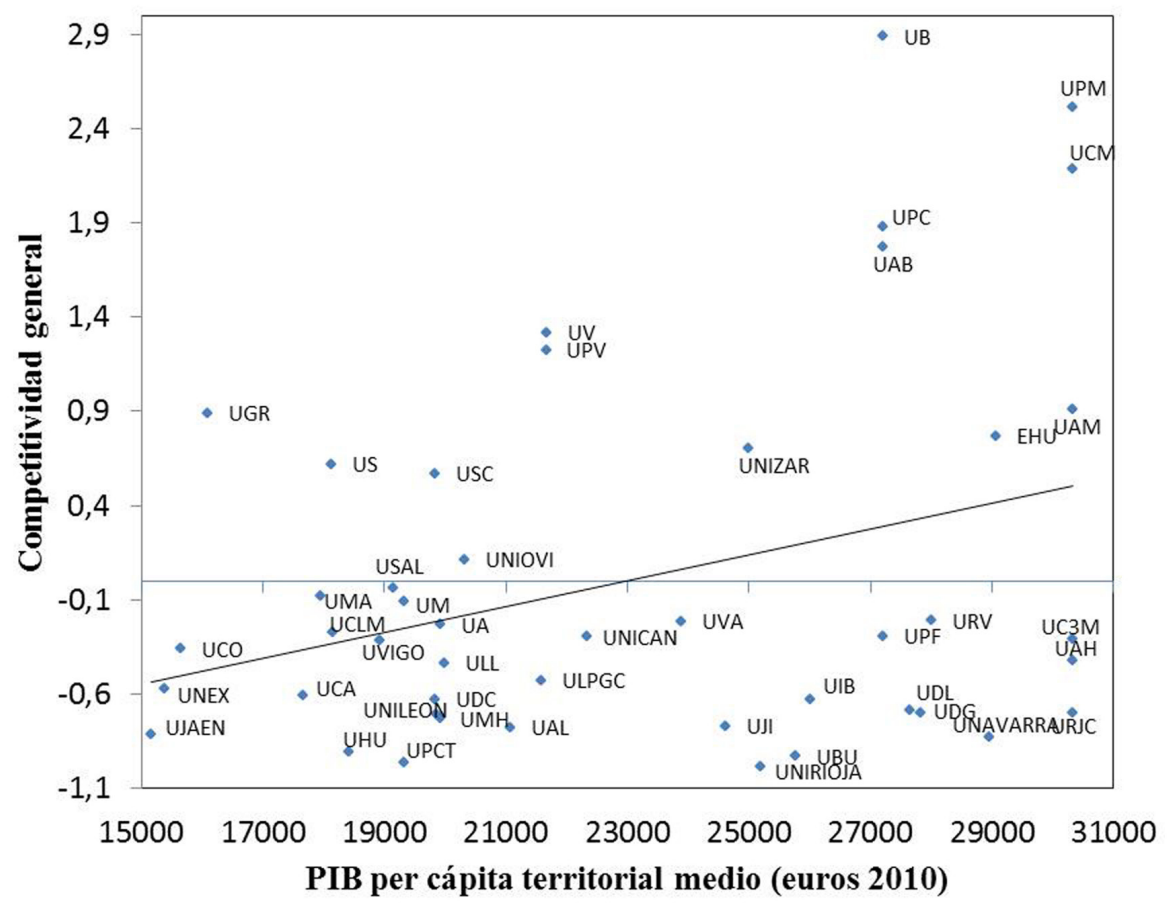

\section{Capacidad formativa}

La última dimensión es la capacidad formativa que comprende diez variables observadas y se refieren al número de becas FPI y FPU, número de contratos Juan de la Cierva y Ramón y Cajal y número de tesis doctorales, todas ellas tanto en valores absolutos como relativos por profesores. El análisis de componentes principales presenta buenos indicadores. El KMO es 0,63, la prueba de esfericidad es significativa y todas las variables consideradas tienen comunalidades muy elevadas (tabla III).

Se obtienen dos componentes que explican algo más del $88 \%$ de la varianza. Como en el caso anterior, se trata de una solución obvia puesto que la primera componente hace referencia a valores absolutos y la segunda a valores relativos por el número de profesores (tabla VII).
La primera componente, "capacidad formativa general", no presenta relación con el PIB pc.

La "capacidad formativa relativa o por profesor", o segunda componente, está correlacionada con el PIB territorial. Efectivamente, hay una relación significativa $(0,03)$ con un $R^{2}=0,10$ y un coeficiente estandarizado beta de 0,32. A mayor PIB pc mayor número de becas (FPI, FPU), mayor número de contratos Ramón y Cajal y Juan de la Cierva y mayor número de tesis doctorales todo ellos relativizados por el número de profesores.

De nuevo el PIB pc marca diferencias. Las universidades en zonas de mayor PIB pc tienen un mayor número de becas, más contratos para personal especializado y mayor número de tesis, todo ello por profesor. En los territorios con mayor PIB pc, figura 5, destaca con mucha diferencia la universidad Pompeu Fabra y después las universidades de Car- 
Tabla VII. Matriz de componentes rotados dimensión "capacidad formativa"

\begin{tabular}{lcc} 
& Componente 1 & Componente 2 \\
\hline FPI_Prom & $\mathbf{0 , 9 3 6}$ & 0,163 \\
\hline FPI_100P_Prom & 0,171 & $\mathbf{0 , 9 1 9}$ \\
\hline FPU_Prom & $\mathbf{0 , 9 7 2}$ & 0,037 \\
\hline FPU_100P_Prom & 0,609 & $\mathbf{0 , 6 3}$ \\
\hline JCierva_Prom & $\mathbf{0 , 8 6 8}$ & 0,369 \\
\hline JCierva_100P_Prom & 0,059 & $\mathbf{0 , 9 5 8}$ \\
\hline RyCajal_Prom & $\mathbf{0 , 8 9 7}$ & 0,319 \\
\hline RyCajal_100P_Prom & 0,105 & $\mathbf{0 , 9 5 9}$ \\
\hline Tesis_Prom & $\mathbf{0 , 9 8 6}$ & $-0,007$ \\
\hline Tesis_100P_Prom & 0,588 & $\mathbf{0 , 5 9 9}$ \\
\hline
\end{tabular}

Método de rotación: Normalización Varimax con Kaiser.

los III y las dos autónomas (Madrid y Barcelona), además de la de Cantabria. En el otro extremo del PIB las que destacan son las universidades Pablo de Olavide, Miguel Hernández, Córdoba y Granada.

\section{Análisis de las diferencias entre universidades de territorios con mayor y menor PIB pc}

Para todas las sub-dimensiones o componentes, se efectúa un análisis de la varianza para averiguar si hay o no diferencias estadísticamente significativas entre las universidades que están en zonas con mayor y menor PIB pc y las diferentes sub-dimensiones de la actividad investigadora. Para formar los grupos se utiliza la mediana del PIB pc, de manera que un grupo está formado por las 23 universidades en zonas que superan la mediana (21.675 euros de 2010) y otro por las 24 que están en zonas por debajo de esa mediana. El primer grupo tiene un PIB pc medio un $44,59 \%$ mayor que el segundo grupo.

Efectivamente, el análisis de la varianza confirma lo comentado para las diferentes dimensiones. Se constata que las universidades en territorios con mayor PIB per cápita tienen mayores niveles de actividad científica por profesor (con un nivel de significación de 0,05), de competitividad, tanto general como por profesor, (nivel de significación 0,01 ) y de capacidad formativa por profesor (nivel de significación 0,03).

Las diferencias más significativas se dan en la competitividad por profesor, aunque es casi igual que el valor de la capacidad formativa general, es decir, en la capacidad para captar proyectos tanto del Plan Nacional como del Programa Marco tanto por profesor como general. Esto es algo fundamental puesto que condiciona otros indicadores de la actividad investigadora. Al fin y al cabo, esto significa mayor captación de recursos directamente orientados a la producción científica, lo que redundará en número de publicaciones, colaboraciones, tesis doctorales o acciones de innovación al mismo tiempo que condiciona la capacidad formativa, puesto que a los proyectos se asocian becas, contratos y tesis doctorales.

Sigue en importancia de significación la capacidad formativa por profesor, esto es el número de becas de FPI y FPU; el número de contratos Juan de la Cierva o Ramón y Cajal y el número de tesis por profesor. Efectivamente, este tipo de becas y contratos es un antecedente de producción científica y de innovación. Por las características y los objetivos que persiguen, una mayor disposición de estas becas y contratos implica mayor producción científica y esto se produce en mayor medida en las universidades de territorios con mayor PIB per cápita.

La actividad científica por profesor sigue en nivel de significación, lo que es razonable de acuerdo con el argumento expuesto.

De nuevo hay que recordar la particularidad de la dimensión de innovación por no disponer de todos los datos. Sin embargo, con la estimación de los datos que faltan, las universidades en zonas de mayor PIB pc tienen más ingresos por contratos de I+D-consultorías y prestación de servicios que las que universidades en zonas de menor PIB, con un nivel de significación del 0,04.

\section{CONCLUSIONES, IMPLICACIONES Y LIMITACIONES}

La actividad investigadora de las universidades se realiza en un contexto económico determinado y dicho contexto no es neutro. Hay una estrecha relación entre la investigación y el entorno que se intenta promover desde instituciones públicas, un buen exponente de esto es la Estrategia Europa 2020 o las estrategias de especialización inteligente por territorios, conocidas como RIS 3.

El presente análisis de la actividad investigadora se realiza distinguiendo entre las diferentes dimen- 
Figura 5. Representación de las universidades públicas españolas según el PIB pc territorial y la dimensión "capacidad formativa por profesor" (los ejes de la figura se han escalado con el fin de ganar en la visibilidad de los puntos)

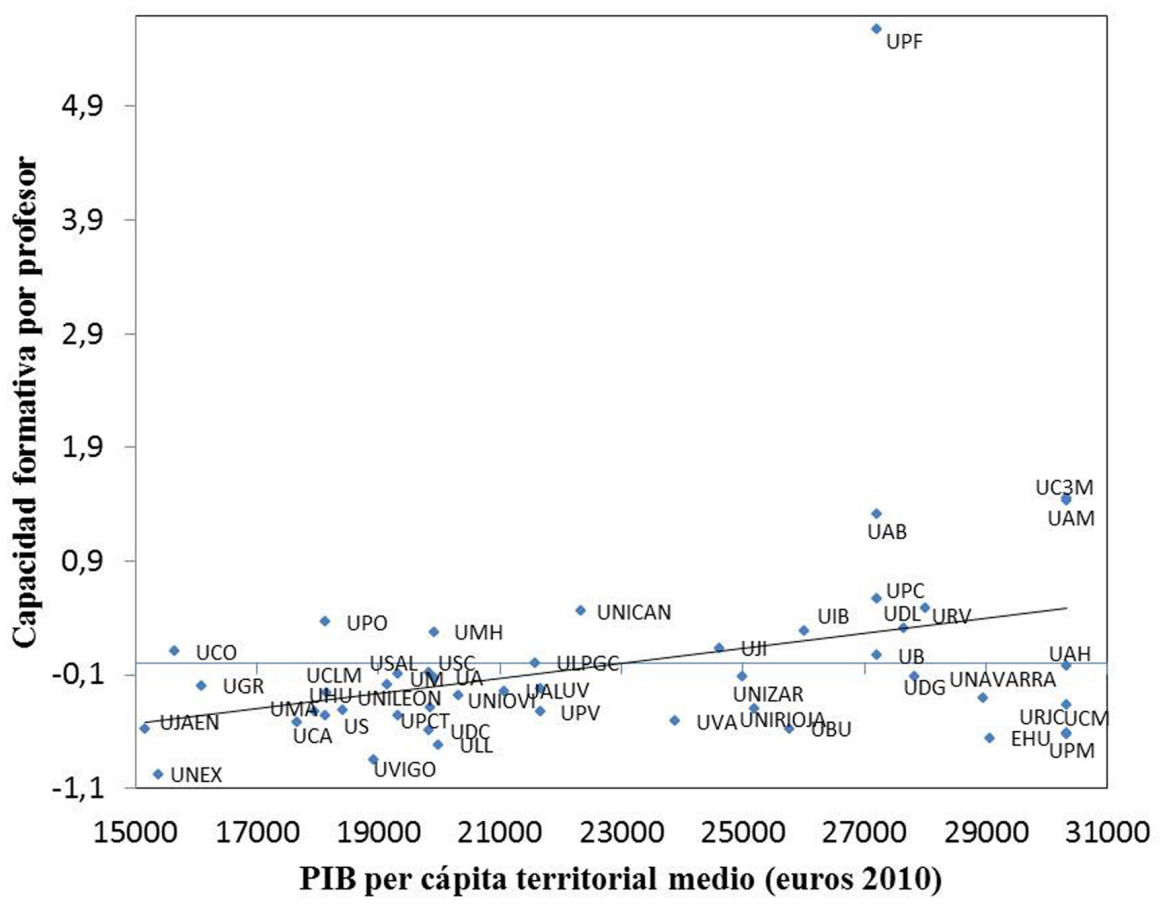

siones de la misma y considerando el contexto económico, medido por el producto interior bruto per cápita del territorio analizado, a partir de lo cual se llega a las siguientes conclusiones.

Efectivamente, el PIB pc del territorio tiene que ver con diferencias en la actividad investigadora, puesto que está correlacionado positivamente con los indicadores de la actividad investigadora. En términos generales, a medida que aumenta el PIB pc del territorio donde están las universidades los indicadores de actividad investigadora son mayores.

Cuando los indicadores de la actividad científica están relativizados o referidos al número de profesores es cuando más fuerte se muestra esta relación, porque se produce en las tres dimensiones, es decir, en actividad científica, en competitividad y en capacidad formativa. Mientras que para los valores generales o en términos absolutos, el PIB pc solamente genera diferencias en competitividad general.

La mayor incidencia del PIB pc se da en la competitividad relativa. Las universidades en territorios con mayor producción económica consiguen mayor número de proyectos, tanto en el ámbito nacional como europeo.

En la dimensión de innovación, como era de esperar, las universidades en territorios con mayor PIB consiguen más ingresos por contratos de la investigación y desarrollo que realizan, las consultorías que efectúan o la prestación de servicios.
En general, las universidades de mayor tamaño no suelen estar en las primeras posiciones en las componentes relativas por número de profesores, independientemente de los niveles de PIB pc. Mantener mejores indicadores por profesor es más difícil con tamaños grandes o a medida que aumenta el tamaño, por tanto en las posibles comparaciones que se establezcan es recomendable tomar en consideración la diferencia por tamaño existente. Por otro lado, la estructura de titulaciones puede afectar, los datos analizados son previos a la implantación de los nuevos grados. Así, una universidad con más titulaciones de primer ciclo (escuelas técnicas o diplomaturas) cuenta con más profesores que no tienen tanta orientación a la investigación y resultaría con peores indicadores al relativizar por profesor. Otro tanto sucede con la composición de las titulaciones por áreas, unas áreas están más orientadas a las patentes o la generación de spin-off que otras, por lo que influirán en los indicadores de resultados.

Para un análisis más detallado considerando la heterogeneidad de las universidades sería interesante tener en cuenta conjuntamente características como tamaño, especialidad o número de estudiantes por profesor, pero no es viable. El número de subconjuntos o grupo resultantes es demasiado elevado para utilizar con el número de universidades disponibles. Aunque la consideración de especialidades ya se ha tenido en cuenta en el análisis de la actividad científica (Bordón y otros, 2010). 
Queda patente que la consideración de sub-dimensiones enriquece y aporta matices interesantes al análisis de la actividad investigadora de las diferentes universidades.

Dentro de niveles de PIB pc es posible identificar universidades que presentan mejores resultados de actividad investigadora. En general, para un nivel de PIB pc alto destaca la Pompeu Fabra además de la universidad Carlos III, mientras que entre las de menor PIB pc destacan la universidad Miguel Hernández, Córdoba y Pablo de Olavide.

Por otro lado y asumiendo el importante papel de la universidad en el desarrollo de su entorno (Smilor y otros, 1993) y en la localización de centros de investigación (Siedschlag y otros, 2013), es preciso introducir la siguiente reflexión: si en todos los territorios se dedicase el mismo porcentaje del PIB a la actividad investigadora persistiría la existencia de desigualdades en la financiación de la misma. Los territorios con mayor PIB destinarían más recursos en términos absolutos que los de menos PIB y a igualdad, o más concretamente dependiendo, del número de estudiantes la financiación sería desigual, esto redunda en un diferente efecto sobre su entorno y en una menor capacidad de atracción de nuevos centros de investigación. En definitiva, es necesario tomar en consideración los recursos con los que finalmente se cuenta. En consecuencia, una futura línea de investigación para completar este análisis es la toma en consideración de los recursos con los que cuenta cada universidad.

De todo lo anterior, se puede deducir que los territorios con un contexto económico más desfavorable están en desventaja respecto a la actividad investigadora universitaria que se realiza en ellos. Esto puede contribuir a un círculo negativo en el que a menor PIB menor investigación, lo que no favorece el crecimiento del PIB y, así, sucesivamente. La dotación de recursos es fundamental para romper esta espiral, pero no solamente alcanzando el porcentaje sobre el PIB medio (lo cual seguiría representando menos recursos para los territorios menos ricos, por comparación con los más ricos), sino que es necesario que ese porcentaje sea mayor. La formación de un sistema de $I+D+i$ eficiente y de un capital humano preparado que sea capaz de atraer recursos, proyectos nacionales o internacionales, es otra actuación prioritaria para la mejora.

Finalmente, es necesario subrayar de nuevo que los datos referidos a los indicadores de innovación son incompletos y sobre esta parte de la actividad investigadora el análisis realizado no permite extraer conclusiones con las mismas garantías. La medida de este tipo de datos viene siendo algo difícil tanto en ámbito nacional como internacional, aunque se han realizado progresos en los últimos años. Seguramente en futuras ediciones se podrá disponer de estadísticas me- jores y más completas. Por otra parte, hay que recordar que se trabaja con sub-dimensiones que sintetizan la mayor parte, que no el total, de la información de los indicadores.

\section{BIBLIOGRAFÍA}

Acs, Z. J.; Anselin, L.;Varga, A. (1997). Local Geographic Spillovers between University Research and High Technology Innovations. Journal of Urban Economics, 42, 422-448. http://dx.doi.org/10.1006/ juec. 1997.2032

Acs, Z. J., ;Anselin, L.; Varga, A. (2002). Patents and innovation counts as measures of regional production of new knowledge. Research Policy, vol. 31, 1069-1085. http://dx.doi.org/10.1016/S00487333(01)00184-6

Bordons, M; Sancho, R.; Morillo, F.; Gómez, I. (2010) Perfil de la actividad científica de las universidades españolas en cuatro áreas temáticas: un enfoque multifactorial. Revista Española de Documentación Científica, 33 (1), 9-33. http://dx.doi.org/10.3989/ redc.2010.1.718

ARWU, Academic Ranking of World Universities (2013). http://www.shanghairanking.com

Etzkowitz, H., \& Leydesdorff, L. (1998). The endless transition: A "triple helix" of university-industrygovernment relations. Minerva vol.36, 203-208. http://dx.doi.org/10.1023/A:1004348123030

Instituto Nacional de Estadística (2012). Contabilidad Regional de España. www.ine.es.

Jaffe, A. B. (1989). Real effects of academic research. American Economic Review, 79 (5), 957-970.

Jöreskog, K.G.; Sörbom, D. (2006). Interactiva LISREL. Online Help File for LISREL 8.8 for Windows. Scientific Software International Inc.

Kim, Y.; Kim, W.; Yang, T. (2012). The effect of the triple helix system and habitat on regional entrepreneurship: Empirical evidence from the U.S.. Research Policy 41 (1) 154-166.

King, D.A. (2004). The scientific impact of nations. Nature, vol. 430 (15), 311-316. http://dx.doi. org/10.1038/430311a

Li, F.;Yi, Y.; Guo, X.; Qi, W. (2012): Performance evaluation of research universities in Mainland China, Hong Kong and Taiwan: based on a two-dimensional approach. Scientometrics, 90:531-542. http:// dx.doi.org/10.1007/s11192-011-0544-1

Lin, X.; Roberts, J. (2012): A stages approach to the internationalization of higher education? The entry of UK universities into China. The Service Industries Journal, vol. 32, (7), 1011-1038. http:// dx.doi.org/10.1080/02642069.2012.662495

Lowry, R.C. (2001). Governmental Structure, Trustee Selection, and Public University Prices and Spending: Multiple Means to Similar Ends. American Journal of Political Science, 45 (4), 845-864. http://dx.doi.org/10.2307/2669328 
Luque-Martínez, T. (2013). La actividad investigadora de la universidad española en la primera década del siglo XXI. Revista Española de Documentación Científica, 36 (4) octubre-diciembre. http://dx.doi. org/10.3989/redc.2013.4.1046

Luque-Martínez, T. (2012). Técnicas de análisis de datos en investigación de mercados. Pirámide. Madrid.

Luque-Martínez, T., Del Barrio-García, S., Aguayo Moral, J.M. (2009). Estudio del impacto económico de la Universidad de Granada en su entorno. Ed. Universidad de Granada. Granada (España).

Medina, F.; Galván, M. (2007). Imputación de datos: teoría y práctica. Estudios estadísticos y prospectiva. CEPAL Santiago de Chile.

NTU National Taiwan University Tanking (2013). http://nturanking.lis.ntu.edu.tw

OECD (2011). OCDE Science, Technology and Industry Scoreboard 2011: Innovation and Growth in Knowledge Economies http://www.oecd.org/ innovation/innovationinsciencetechnologyandindustry/oecdsciencetechnologyandindustryscoreboard2011innovationandgrowthinknowledgeeconomies.htm

Olssen, M.; Peters, M. (2005): Neoliberalism, higher education and the knowledge economy: from the free market to knowledge capitalism. Journal of Education Policy, vol. 20, (3), 313-345. http:// dx.doi.org/10.1080/02680930500108718

RIS 3 (2013). Guide to Research and Innovation Strategies for Smart Specialisation (RIS 3) 2013. http://s3platform.jrc.ec.europa.eu/en/c/document library/get file?uuid=e50397e3-f2b1-40868608-7b86e69e8553

Sanz-Casado. E. (2012). Lanzamiento del Observatorio IUNE, una herramienta para el seguimiento de la actividad científica de las universidades españolas. Revista Española de Documentación Científica, vol. 35 (3), 503-505, 2012. ISSN: 0210-0614.
Sanz-Casado, E.; García-Zorita, C; Serrano-López, A.E.; Efraín-García. P.; De Filippo, D. (2013). Rankings nacionales elaborados a partir de múltiples indicadores frente a los de índices sintéticos. Revista Española de Documentación Científica, vol. 36 (3), 1-18.

Siedschlag, I.; Smith, D.; Turcu, C.; Zhang, X. (2013). What determines the location choice of R\&D activities by multinational firms? Research Policy 42 (8) 1420-1430. http://dx.doi.org/10.1016/j.respol.2013.06.003

Smilor, R. W.; Dietrich, G. B.; Gibson, D. V. (1993). The entrepreneurial university-the role of highereducation in the United-States in technology commercialization and economic-development. International Social Science Journal, 45(1), 1-11.

Stiglitz, J.E.; Sen, A.; Fitoussi, J.P. (2013). Medir nuestras vidas. Las limitaciones del PIB como indicador de progreso. Ed. RBC.

Sun, Y.; Liu, F. (2012) Measuring international traderelated technology spillover: a composite approach of network analysis and information theory. Scientometrics $\mathrm{http}: / / \mathrm{dx}$.doi.org/10.1007/s11192-0120860-0

Tijssen, R.J.W. (2004). Is the commercialisation of scientific research affecting the production of public knowledge? Global trends in the output of corporate research articles. Research Policy 33, 709-733. http://dx.doi.org/10.1016/j.respol.2003.11.002

THE, Times Higher Education (2013). http://www. timeshighereducation.co.uk/world-universityrankings

Vincett, P.S. (2010) The economic impacts of academic spin-off companies, and their implications for public policy. Research Policy 39 (6) 737-747. http://dx.doi.org/10.1016/j.respol.2010.02.001 
ANEXO 1. Relación de universidades públicas presenciales españolas con la provincia o Comunidad Autónoma de referencia para el PIB, antigüedad, tamaño y el PIB per cápita de su territorio

\begin{tabular}{|c|c|c|c|c|c|}
\hline Universidad & Acrónimo & $\begin{array}{l}\text { Provincia/Comunidad } \\
\text { Autónoma de referen- } \\
\text { cia para el PIB }\end{array}$ & $\begin{array}{l}\text { Antigüedad } \\
\text { (años) }\end{array}$ & $\begin{array}{l}\text { No medio de } \\
\text { profesores }\end{array}$ & $\begin{array}{l}\text { PIB pc medio } \\
\text { (euros 2010) }\end{array}$ \\
\hline País Vasco & EHU & País Vasco & 44 & 2.474 & 29.075 \\
\hline Alicante & UA & Alicante & 33 & 990 & 19.916 \\
\hline Autónoma de Barcelona & UAB & Barcelona & 44 & 1.446 & 27.219 \\
\hline Alcalá de Henares & $\mathrm{UAH}$ & Madrid & 35 & 830 & 30.337 \\
\hline Almería & UAL & Almería & 19 & 513 & 21.080 \\
\hline Autónoma de Madrid & UAM & Madrid & 44 & 1.444 & 30.337 \\
\hline Barcelona & UB & Barcelona & 582 & 2.687 & 27.219 \\
\hline Burgos & UBU & Burgos & 18 & 376 & 25.764 \\
\hline Carlos III & UC3M & Madrid & 23 & 473 & 30.337 \\
\hline Cádiz & UCA & Cádiz & 33 & 884 & 17.660 \\
\hline Castilla-LaMancha & UCLM & Castilla-LaMancha & 30 & 980 & 18.161 \\
\hline Complutense Madrid & UCM & Madrid & 719 & 3.816 & 30.337 \\
\hline Córdoba & UCO & Córdoba & 40 & 803 & 15.651 \\
\hline Coruña (La) & UDC & La Coruña & 23 & 839 & 19.840 \\
\hline Gerona & UDG & Gerona & 21 & 438 & 27.832 \\
\hline Lérida & UDL & Lérida & 21 & 424 & 27.647 \\
\hline Granada & UGR & Granada & 481 & 2.246 & 16.085 \\
\hline Huelva & UHU & Huelva & 19 & 419 & 18.429 \\
\hline Islas Baleares & UIB & Islas Baleares & 34 & 524 & 26.012 \\
\hline Jaén & UJAEN & Jaén & 19 & 575 & 15.171 \\
\hline Jaume I & UJI & Castellón & 21 & 456 & 24.620 \\
\hline La Laguna & ULL & Tenerife & 220 & 1.311 & 19.992 \\
\hline Las Palmas de GC & ULPGC & Las Palmas & 23 & 938 & 21.591 \\
\hline Murcia & UM & Murcia & 97 & 1.235 & 19.335 \\
\hline Málaga & UMA & Málaga & 40 & 1.352 & 17.958 \\
\hline Miguel Hernández & UMH & Alicante & 16 & 349 & 30.337 \\
\hline Navarra & UNAVARRA & Navarra & 25 & 384 & 28.959 \\
\hline Extremadura & UNEX & Extremadura & 39 & 1.013 & 15.381 \\
\hline Cantabria & UNICAN & Cantabria & 40 & 639 & 22.340 \\
\hline León & UNILEON & León & 33 & 622 & 19.868 \\
\hline Oviedo & UNIOVI & Asturias & 404 & 1.491 & 20.332 \\
\hline Rioja (La) & UNIRIOJA & Rioja (La) & 20 & 263 & 25.200 \\
\hline Zaragoza & UNIZAR & Aragón & 538 & 1.725 & 25.006 \\
\hline Politécnica de Cataluña & UPC & Barcelona & 41 & 1.388 & 27.219 \\
\hline Politécnica de Cartagena & UPCT & Murcia & 14 & 293 & 19.335 \\
\hline Pompeu Fabra & UPF & Barcelona & 22 & 285 & 27.219 \\
\hline Politécnica de Madrid & UPM & Madrid & 41 & 2.481 & 30.337 \\
\hline Pablo de Olavide & UPO & Sevilla & 14 & 184 & 18.131 \\
\hline Politécnica de Valencia & UPV & Valencia & 41 & 1.750 & 21.675 \\
\hline Rey Juan Carlos & URJC & Madrid & 16 & 622 & 30.337 \\
\hline Rovira i Virgili & URV & Tarragona & 21 & 509 & 28.012 \\
\hline
\end{tabular}




\begin{tabular}{lllccc}
\hline Universidad & Acrónimo & $\begin{array}{l}\text { Provincia/Comunidad } \\
\text { Autónoma de referen- } \\
\text { cia para el PIB }\end{array}$ & $\begin{array}{c}\text { Antigüedad } \\
\text { (años) }\end{array}$ & $\begin{array}{c}\text { No medio de } \\
\text { profesores }\end{array}$ & $\begin{array}{c}\text { PIB pc medio } \\
\text { (euros 2010) }\end{array}$ \\
\hline Sevilla & US & Sevilla & 507 & 2.398 & 18.131 \\
\hline Salamanca & USAL & Salamanca & 794 & 1.366 & 19.157 \\
\hline Santiago de Compostela & USC & La Coruña & 517 & 1.545 & 19.840 \\
\hline Valencia & UV & Valencia & 512 & 2.248 & 21.675 \\
\hline Valladolid & UVA & Valladolid & 717 & 1.443 & 23.891 \\
\hline Vigo & UVIGO & Pontevedra & 23 & 952 & 18.930 \\
\hline
\end{tabular}

